\section{Myeloablative conditioning using timed-sequential busulfan plus fludarabine in older patients with acute myeloid leukemia: long-term results of a prospective phase II clinical trial}

Myeloablative hematopoietic cell transplantation (HCT) reduces relapse, but is precluded in older patients and those with high comorbidities in whom outcomes remain disappointing. After demonstrating the safety of timed-sequential administration of myeloablative busulfan with fludarabine (Bu-Flu) in older patients and those with high comorbidities, ${ }^{1}$ we evaluated its efficacy in acute myeloid leukemia (AML). Our original study (clinicaltrials.gov identifier: 01572662) was an equally randomized Phase II trial that compared two myeloablative timed-sequential Bu-Flu conditioning regimens: lower dose busulfan [area under the curve (AUC) $=16,000 \pm 12 \% \mu \mathrm{mol} / \mathrm{min}$, the $16 \mathrm{~K}$ arm] versus higher dose (AUC=20,000 $\pm 12 \% \mu \mathrm{mol} / \mathrm{min}$, the $20 \mathrm{~K} \mathrm{arm}$ ), as described previously. ${ }^{1}$ Graft-versus-host disease (GvHD) prophylaxis included tacrolimus and methotrexate. ${ }^{1}$ All patients who met the eligibility criteria and were deemed suitable for the trial by treating physicians were enrolled. After 98 patients were enrolled, the randomization was stopped because the $20 \mathrm{~K}$ arm was demonstrated to be as safe as the $16 \mathrm{~K}$ arm. The outcomes of those patients were reported. ${ }^{1}$ To estimate the efficacy of this regimen, the trial was extended and 101 additional patients with hematologic malignancies were enrolled onto the higher dose (20K) arm with the approval of the institutional review board. Herein, we report the outcomes of AML patients $(n=71)$. The research was conducted in accordance with the Declaration of Helsinki and all participants provided written informed consent.

The primary outcome was efficacy, as determined by the rates of relapse, progression-free survival (PFS; defined as the time from HCT to relapse/progression or death) and overall survival (OS; defined as the time from HCT to death from any cause). Categorical variables were compared between treatment arms using Fisher's exact test. Continuous variables were compared using the Wilcoxon rank sum test. The rate of non-relapse mortality (NRM) was estimated in a competing risks framework, with relapse as the competing risk. Acute and chronic GvHD were assessed with competing risks of relapse and death. Kaplan-Meier curves were used to estimate OS and PFS, and the log-rank test was used to test differences between groups based on variables of interest. Cox proportional hazards regression models were fit to model the association between OS, PFS, relapse, and NRM and co-variates of interest. Results were analyzed by pre-HCT disease status as that is one of the most important factors predicting post-HCT outcomes. All statistical analyses were performed using $\mathrm{R}$ version 3.5.1.

Seventy-one patients with AML were enrolled. Table 1 summarizes the patients' characteristics. Median age was 64 years (range 29-73 years). A majority had matched unrelated donors $(n=45,63 \%)$ and received peripheral blood grafts $(n=46,65 \%)$. Most patients $(n=59,83 \%)$ received busulfan $20 \mathrm{~K}$ (median, $12.7 \mathrm{mg} / \mathrm{kg}$ intravenously; interquartile range (IOR) 10.79-14.02]; the rest received 16K (median, $9.7 \mathrm{mg} / \mathrm{kg}$ intravenously; IOR 8.53-10.74). Over 60\% had primary induction failure (PIF; $n=32$ ) or relapsed disease $(n=11)$. Nearly half $(n=33,47 \%)$ had adverse-risk disease as per the European LeukemiaNet (ELN) revised classification. ${ }^{2}$ Fifty-five percent had HCT comorbidity index (HCT-CI) score $>3.3$ The median follow up was 40 months (IOR: 34.11-48.69) among survivors.

There were no graft failures. All patients engrafted neutrophils (absolute neutrophil count of $>0.5 \times 10^{9} / \mathrm{L}$ for 3 con- secutive days) at a median of 12 days (IOR: 11-15). Of the 71 patients, 66 engrafted platelets $\left(>20 \times 10^{9} / \mathrm{L}\right.$ without transfusion for 7 consecutive days) at a median of 14 days (IOR: 11-19). At day 30, whole-blood chimerism analysis showed a median of $100 \%$ (IOR: $98-100 \%$ ) cells of donor origin. The median T-cell chimerism was $85 \%$ (IOR: $70-100 \%$ ) at day 30 , which increased to $96 \%$ (IOR: $82-100 \%$ ) at day 100 , and $100 \%$ (IOR: $100-100 \%$ ) by one year. In the myeloid compartment, we observed $100 \%$ donor cells throughout the study period. The cumulative incidences of grade II-IV and III-IV acute GvHD at day 100 were 39\% [95\% confidence interval (CI): $28-51 \%$ ] and $10 \%(95 \% \mathrm{CI}$ :

Table 1. Baseline patients' characteristics.

\begin{tabular}{lc} 
Characteristic & $\begin{array}{c}\text { Number of patients } \\
n=71\end{array}$ \\
Age at HCT in years, median (IQR) & $64(57-69)$ \\
Males, n (\%) & $43(61)$ \\
Race, n (\%) & \\
White & $59(83)$ \\
Other & $12(17)$ \\
Diagnosis, n (\%) & \\
Primary AML & $51(72)$ \\
Secondary AML (MDS/MPD) & $20(28)$ \\
\hline Donor, n (\%) & \\
HLA-matched, unrelated & $45(63)$ \\
HLA-matched sibling & $26(37)$ \\
Graft source, n (\%) & \\
PB & $46(65)$ \\
BM & $25(35)$
\end{tabular}

Busulfan dose, n (\%)

$\begin{array}{ll}\text { AUC 20K dose } & 59(83) \\ \text { AUC 16K } & 12(17)\end{array}$

Disease status, ${ }^{*} \mathrm{n}(\%)$

CR 1/2 (MRD negative)

CR 1/2 (MRD positive)

Primary induction failure

Relapsed disease

Revised ELN classification, $\mathrm{n}(\%)$

$\begin{array}{lc}\text { Favorable } & 8(11) \\ \text { Intermediate } & 30(42) \\ \text { Adverse } & 33(47)\end{array}$

HCT-CI, n (\%)

$0-2$

$\geq 3$

Follow-up in months, median (IQR) $40(34-49)$

* One patient in complete remission (CR) did not have minimal residual disease (MRD) analysis performed before hematopoietic cell transplantation (HCT). MRD assessment was performed by either multi-parameter flow cytometry (MFC) or quantitative polymerase chain reaction (PCR). Of the $27 \mathrm{MRD}$ positive patients, 18 were positive by both MFC and PCR, and nine were positive only by PCR. Among 18 patients who were MRD positive by MFC, 11 patients were $1 \%$ or higher, two patients were $<0.1 \%$ and the rest were between $0.1-1 \%$. CR is defined as the morphological complete remission with $<5 \%$ blasts. *All patients were retrospectively categorized as per the Revised European LeukemiaNet (ELN) classification.AML: acute myeloid leukemia; AUC: area under the curve; BM: bone marrow; CR1: first complete remission; CR2: second complete remission; HCT: hematopoietic cell transplantation; HCT-CI: hematopoietic cell transplantation-specific comorbidity index; HLA: human leukocyte antigen; IQR: interquartile range; MDS: myelodysplastic syndromes; MPD: myeloproliferative disorder; MRD: measurable residual disease; PB peripheral blood. 
Table 2. Results of multivariate analyses.

\begin{tabular}{|c|c|c|c|c|c|c|c|c|c|c|c|c|}
\hline & & OS & & & PFS & & & Relapse & & & NRM & \\
\hline & HR & $95 \%$ CI & $P$ & HR & $95 \%$ CI & P & HR & $95 \%$ Cl & $\mathbf{P}$ & HR & $95 \%$ CI & $\mathbf{P}$ \\
\hline Busulfan dose & & & & & & & & & & & & \\
\hline $16 \mathrm{~K}$ & 1 & & & 1 & & & 1 & & & 1 & & \\
\hline $20 \mathrm{~K}$ & 0.89 & $0.35-2.24$ & 0.79 & 0.79 & $0.33-1.94$ & 0.62 & 0.39 & $0.18-0.86$ & 0.02 & 1.98 & $0.49-7.89$ & 0.33 \\
\hline Age at HCT & & & & & & & & & & & & \\
\hline$<60$ years & 1 & & & 1 & & & 1 & & & 1 & & \\
\hline$>60$ years & 1.24 & $0.63-2.45$ & 0.53 & 1.04 & $0.55-1.98$ & 0.90 & 0.88 & $0.41-1.87$ & 0.73 & 2.26 & $0.79-6.47$ & 0.13 \\
\hline Disease status at $\mathrm{HC}$ & & & & & & & & & & & & \\
\hline CR/MRD-negative & 1 & & & 1 & & & 1 & & & & & \\
\hline Others & 2.67 & $0.99-7.23$ & 0.05 & 3.22 & $1.27-8.15$ & 0.01 & 2.29 & $0.77-6.85$ & 0.14 & - & - & - \\
\hline ELN disease risk & & & & & & & & & & & & \\
\hline Favorable & 1 & & & 1 & & & 1 & & & & & \\
\hline Intermediate & 1.14 & $0.32-4.11$ & 0.84 & 0.79 & $0.25-2.49$ & 0.69 & 1.94 & $0.23-16.28$ & 0.54 & - & - & - \\
\hline Adverse & 1.74 & $0.49-6.10$ & 0.39 & 1.36 & $0.45-4.11$ & 0.58 & 4.9 & $0.57-41.65$ & 0.15 & - & - & - \\
\hline HCT-CI & & & & & & & & & & & & \\
\hline $0-2$ & 1 & & & 1 & & & & & & 1 & & \\
\hline$>3$ & 1.98 & $1.00-3.91$ & 0.05 & 1.29 & 0.69-2.42 & 0.42 & - & - & - & 3.97 & 1.41-11.14 & 0.009 \\
\hline
\end{tabular}

CI: Confidence Interval; ELN: European LeukemiaNet; HCT: hematopoietic cell transplantation; HCT-CI: hematopoietic cell transplantation-specific comorbidity index; HR: Hazard Ratio; OS: overall survival; PFS: progression-free survival; CR: complete remission; NRM: non-relapse mortality. MRD: minimal residual disease.

3-17\%), respectively. The cumulative incidences of overall chronic GvHD and systemic therapy-requiring chronic GvHD were both 20\% (95\%CI: $10-29 \%$ ) at one year.

The cumulative incidence of relapse was $34 \%(95 \% \mathrm{CI}$ : $23-45 \%)$ at two years; it was $11 \%(95 \% \mathrm{CI}: 0-26 \%)$ in those who were in complete remission (CR) with undetectable measurable residual disease (MRD) versus 42\% (95\% CI: 29 $56 \%$ ) in others. In multivariate analysis, the receipt of higher-dose (20K) versus lower-dose (16K) busulfan was the only factor associated with a significantly lower risk of relapse [hazard ratio (HR) 0.39; 95\%CI: 0.18-0.86; $P=0.02$ ] (Table 2). The cumulative incidence of NRM was $6 \%(95 \% \mathrm{CI}: 0$ $11 \%$ ) at day 100 and $24 \%$ (95\%CI: $14-34 \%$ ) at two years. In multivariate analysis, $\mathrm{HCT}-\mathrm{CI}>3$ was the only significant predictor of NRM (HR, 3.97; 95\%CI: $1.41-11.14 ; P=0.009)$ (Table 2). The cumulative incidence of NRM at two years was 33\% (95\% CI: $18-49 \%)$ in patients with $\mathrm{HCT}-\mathrm{CI}>3$ versus $13 \%$ (95\%CI: $8-24 \%)$ in those with HCT-CI $<3 ; P=0.03$.

At two years, the estimated OS was $45 \%$ (95\%CI: $35-$ $58 \%$ ); it was $78 \%$ (95\%CI: 61-100\%) in the CR/MRD-negative group versus 35\% (95\% CI: $24-50 \%$ ) in others (Figure 1A). In multivariate analysis, not being in a CR/MRD-negative state (HR: 2.67; 95\%CI: 0.99-7.23; $P=0.05)$ and having an HCT-CI >3 (HR, 1.98; 95\%CI: 1.00-3.91; $P=0.05)$ were independently associated with a higher risk of mortality (Table 2). At two years, the estimated PFS was $42 \%$ (95\%CI: $32-55 \%$ ); it was $78 \%$ (95\% CI: $61-100 \%$ ) in the CR/MRD-negative group versus 31\% (95\%CI: $21-46 \%$ ) in others (Figure 1B). In multivariate analysis, not being in CR/MRD-negative state at the time of HCT was the only factor associated with a significantly inferior PFS (HR, 3.22; 95\%CI: 1.27-8.15; $P=0.01$ ) (Table 2). Sixteen patients received maintenance with azacytidine $(n=14)$ or FLT-3 inhibitor $(n=2)$. We found no difference in OS among those who received maintenance (median 43 months; 95\%CI: 18-non-analyzable) and those who did not (median 16 months; 95\%CI: 11- non-analyzable), $P=0.23$.

We demonstrated that myeloablative HCT can be safely and effectively performed in older AML patients and those with significant comorbidities by administering busulfan in a timed-sequential manner and closely monitoring the dose through pharmacokinetic analysis. Prior registry study of older AML patients ( $>60$ years) in CR1 showed 2-year PFS and OS of approximately $30-35 \%,{ }^{4}$ and a prospective Phase II trial of AML patients in CR1 (>60 years) showed somewhat better outcomes with a PFS of $42 \%$ and OS of $48 \%$ at two years. ${ }^{5}$ In our study, CR patients (CR1 and CR2) had a 2 -year PFS of $59 \%$ and OS of $67 \%$. Patients who attained MRD-negative CR before HCT had a striking 3-year OS of $78 \%$, which is similar to that of previous reports for younger patients ( $<50$ years) with CR/MRD-negative disease status (2-3 year OS, 73-80\%). ${ }^{6-9}$ Patients with persistent disease at HCT (MRD-positive CR, relapsed disease, or PIF) were analyzed as a single group as they have similarly poor outcomes, as shown by others ${ }^{6}$ and noted by us. The 3-year OS of this group was 33\% (95\% CI: $22-48 \%$ ), which is harmonious with prior reports that showed a 2-3-year OS of approximately $25-30 \%$, regardless of the conditioning intensity, ${ }^{6,9-13}$ and which is comparable to the OS of younger patients (median age $<50$ years) with active disease. ${ }^{13}$

In multivariate analyses, we found that higher myeloablative dose of busulfan (20K) was associated with an approximately $60 \%$ reduced risk of relapse compared to lower dose (16K) but was not a significant predictor of NRM. Notably, age ( $<60$ vs. $>60$ years) was also not a predictor of NRM suggesting that myeloablative doses of busulfan could be safely delivered in a timed-sequential manner across the study population. The only factor associated with high NRM (almost 4-times greater risk) and poor survival (about 2-times greater risk) was an HCT-CI $>3$. However, even in that subgroup, NRM (33\% at 3 years) was not higher than the reported NRM in younger patients with high comorbidities (32-46\% at 2 years) with either myeloablative ${ }^{14}$ or reduced intensity conditioning (RIC). ${ }^{15}$

Our study is limited by a relatively fewer number of patients in CR pre-HCT and a predominance of patients with PIF and relapsed disease. Next, although we demonstrated that higher-dose busulfan was well tolerated and led to lower relapse risk than lower-dose busulfan, how it contrasts directly against other regimens is unknown. Also, we 
A

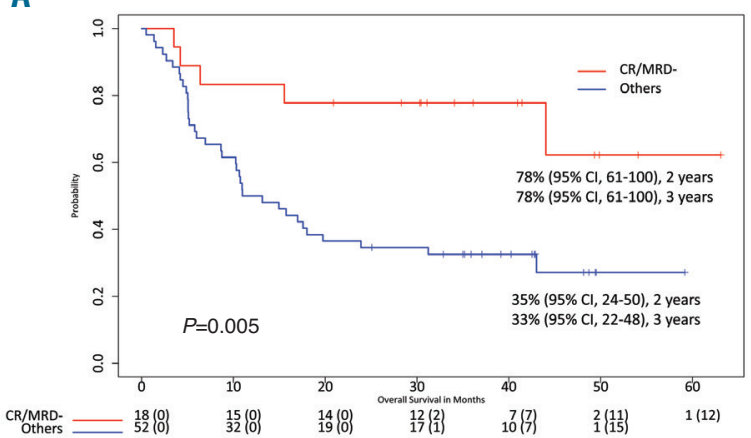

B

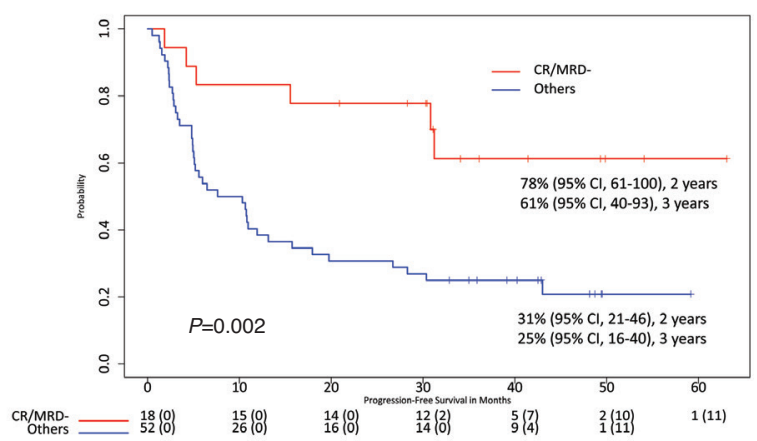

Figure 1. Kaplan-Meier curves showing the impact of pre-hematopoietic cell transplantation (HCT) disease status on survival. (A) Overall survival and (B) progression-free survival; $\mathrm{Cl}$ : Confidence Interval; $\mathrm{CR}$ : complete remission; MRD: measurable residual disease.

categorized patients in CR by the presence or absence of MRD, a well-recognized prognostic factor, but analyzed CR1 and CR2 patients together due to small numbers, which may have deflated our outcomes, as CR2 patients have worse outcomes than those in CR $1 .{ }^{12}$ Lastly, the MRD assessment was conducted using either multi-parameter flow cytometry or quantitative polymerase chain reaction.

Myeloablative conditioning using timed-sequential delivery of busulfan along with fludarabine is safe and effective in older AML patients up to age 73 years and in those with high comorbidities. In an extremely high-risk population, in which over $60 \%$ had PIF or relapsed disease without achieving CR prior to HCT, $55 \%$ had HCT-CI $>3$ and around half had adverse risk disease, our outcomes are encouraging, especially in those who are in remission at the time of HCT. This regimen merits further investigation and comparison to other regimens in older patients with AML.

Rohtesh S. Mehta, ${ }^{1}$ Roland Bassett, ${ }^{2}$ Amanda Olson, Julianne Chen, ${ }^{1}$ Sairah Ahmed, Amin M. Alousi,

Paolo Anderlini, ${ }^{1}$ Gheath Al-Atrash, ${ }^{1}$ Qaiser Bashir,

Stefan O. Ciurea, ${ }^{1}$ Chitra M. Hosing, ${ }^{1}$ Jin S. Im, ${ }^{1}$

Partow Kebriaei, ${ }^{1}$ Issa Khouri, ${ }^{1}$ David Marin, ${ }^{1}$

Jeffrey J. Molldrem, ${ }^{1}$ Yago Nieto, ${ }^{1}$ Betul Oran, ${ }^{1}$

Katayoun Rezvani, Muzaffar H. Qazilbash,

Samer A. Srour, Elizabeth J. Shpall, Borje S. Andersson, ${ }^{1}$

Richard E. Champlin' and Uday R. Popat'

${ }^{1}$ Department of Stem Cell Transplantation and Cellular Therapy and ${ }^{2}$ Department of Biostatistics, The University of Texas MD
Anderson Cancer Center, Houston, TX, USA

Acknowledgments: we acknowledge Laura Russell from The

University of Texas MD Anderson Cancer Center's Department

of Scientific Publications for editorial assistance.

Funding: this work was supported in part by the Cancer Center

Support Grant (NCI Grant P30 CA016672).

Correspondence: UDAY POPAT

upopat@mdanderson.org

doi:10.3324/haematol.2018.214429

Information on authorship, contributions, and financial \& other disclosures was provided by the authors and is available with the online version of this article at www. haematologica.org.

\section{References}

1. Popat UR, Mehta RS, Bassett R, et al. Fludarabine with a higher versus lower dose of myeloablative timed-sequential busulfan in older patients and patients with comorbidities: an open-label, non-stratified, randomised phase 2 trial. Lancet Haematol. 2018;5(11):e532e542.

2. Dohner H, Estey E, Grimwade D, et al. Diagnosis and management of AML in adults: 2017 ELN recommendations from an international expert panel. Blood. 2017;129(4):424-447.

3. Sorror ML, Maris MB, Storb R, et al. Hematopoietic cell transplantation (HCT)-specific comorbidity index: a new tool for risk assessment before allogeneic HCT. Blood. 2005;106(8):2912-2919.

4. McClune BL, Weisdorf DJ, Pedersen TL, et al. Effect of age on outcome of reduced-intensity hematopoietic cell transplantation for older patients with acute myeloid leukemia in first complete remission or with myelodysplastic syndrome. J Clin Oncol. 2010;28(11):1878-1887.

5. Devine SM, Owzar K, Blum W, et al. Phase II Study of Allogeneic Transplantation for Older Patients With Acute Myeloid Leukemia in First Complete Remission Using a Reduced-Intensity Conditioning Regimen: Results From Cancer and Leukemia Group B 100103 (Alliance for Clinical Trials in Oncology)/Blood and Marrow Transplant Clinical Trial Network 0502. J Clin Oncol. 2015;33(35):4167-4175.

6. Araki D, Wood BL, Othus M, et al. Allogeneic Hematopoietic Cell Transplantation for Acute Myeloid Leukemia: Time to Move Toward a Minimal Residual Disease-Based Definition of Complete Remission? J Clin Oncol. 2016;34(4):329-336.

7. Kanakry CG, O'Donnell PV, Furlong T, et al. Multi-institutional study of post-transplantation cyclophosphamide as single-agent graft-versus-host disease prophylaxis after allogeneic bone marrow transplantation using myeloablative busulfan and fludarabine conditioning. J Clin Oncol. 2014;32(31):3497-3505

8. Walter RB, Buckley SA, Pagel JM, et al. Significance of minimal residual disease before myeloablative allogeneic hematopoietic cell transplantation for AML in first and second complete remission. Blood. 2013;122(10):1813-1821.

9. Walter RB, Gooley TA, Wood BL, et al. Impact of pretransplantation minimal residual disease, as detected by multiparametric flow cytometry, on outcome of myeloablative hematopoietic cell transplantation for acute myeloid leukemia. J Clin Oncol. 2011;29(9):1190-1197.

10. Pohlen M, Groth C, Sauer T, et al. Outcome of allogeneic stem cell transplantation for AML and myelodysplastic syndrome in elderly patients (60 years). Bone Marrow Transplant. 2016;51(11):1441-1448.

11. Han Sy, Romee R, Slade M, et al. Reduced Intensity Hematopoietic Cell Transplantation in Active Disease AML Is Associated with Leukemia Free Survival and Relapse Comparable to Myeloablative Conditioning. Blood. 2016;128(22):3477.

12. Bansal D, Bhamidipati PK, Edwin NC, et al. Post-Transplant Outcomes in AML Patients $\geq 60$ Years of Age Beyond CR1. Blood. 2016;128(22):4696.

13. Weisdorf DJ, Millard HR, Horowitz MM, et al. Allogeneic transplantation for advanced acute myeloid leukemia: The value of complete remission. Cancer. 2017;123(11):2025-2034.

14. Sorror ML, Sandmaier BM, Storer BE, et al. Comorbidity and disease status based risk stratification of outcomes among patients with acute myeloid leukemia or myelodysplasia receiving allogeneic hematopoietic cell transplantation. J Clin Oncol. 2007;25(27):4246-4254.

15. Lim ZY, Ingram W, Brand R, et al. Impact of pretransplant comorbidities on alemtuzumab-based reduced-intensity conditioning allogeneic hematopoietic SCT for patients with high-risk myelodysplastic syndrome and AML. Bone Marrow Transplant. 2010;45(4):633-639. 